\title{
NPR Lenses: Interactive Tools for Non-Photorealistic Line Drawings
}

\author{
Petra Neumann, Tobias Isenberg, and Sheelagh Carpendale \\ Department of Computer Science, University of Calgary, Canada \\ \{pneumann $\mid$ isenberg $\mid$ sheelagh\}@cpsc.ucalgary.ca
}

\begin{abstract}
NPR Lenses is an interactive technique for producing expressive non-photorealistic renderings. It provides an intuitive visual interaction tool for illustrators, allowing them to seamlessly apply a large variety of emphasis techniques. Advantages of $3 \mathrm{D}$ scene manipulation are combined with the capabilities of viewer-aligned lenses by inserting $2 \mathrm{D}$ lens controls into the $3 \mathrm{D}$ rendering pipeline. By using lenses that are spatially explicit and constrained we enable object-independent adjustments in 3D. Regions of emphasis can be easily created by adjusting lens parameters and any combination of line style, type, shape, and position of computer-generated lines. NPR Lenses support the creation of effects similar to those found in many existing hand-drawn illustrations and allow creative freedom through interactive control at the same time.
\end{abstract}

\section{Introduction}

For hundreds of years, humans have been creating illustrations by hand for scientific, medical, technical, artistic, and entertainment purposes. Non-Photorealistic Rendering (NPR, $[1,2]$ ) research deals with the computer-based development of techniques to create such illustrations. While now a large number of NPR techniques exist, they have not yet been widely adopted by the illustration community. NPR research tends to focus on the difficult technical problems that can make these techniques viable. In contrast, few NPR techniques have been developed which provide creative freedom for the illustrator using them. For example, they tend to provide access to a single technique at a time, while many hand-drawn illustrations often incorporate several style variations within a single drawing. The development of such interactive tools is necessary to help illustrators leverage NPR techniques more frequently and successfully. We address this problem of creating tools to support illustrators and present an interactive tool called NPR Lenses that allows artists and illustrators to interactively apply and explore emphasis effects to line drawings similar to those in hand-drawings.

Recent developments in computer-based techniques for line drawings and other NPR methods allow artists to automatically create illustrations. However, many illustrative and expressive techniques common in hand-drawn illustrations remain unexplored in electronic systems. For instance, emphasis has been used over many centuries in art, cartography, and illustration as a means of giving importance and guiding attention. Most hand-made illustrations provide this 
emphasis by adjusting one or more of many different aspects of the illustration of an object such as shape, lighting, style, etc. Such adjustments perform important functions such as conveying the intended meaning, implying relative importance of certain parts, providing room for additional information, and creating humor. These techniques may use variant lines styles and weights, spatial distortion for exaggeration (as in caricatures), or even selective inclusion or exclusion of lines to reveal hidden or internal information. Many of these emphasis techniques have already received research attention, however, previous solutions often (1) have a global effect (on the entire image): illustrators may want explicit control of how the emphasis technique spreads into the rest of the image; (2) create discontinuities: illustrators, in part, create their desired impact by how emphasis in one region is integrated with the rest of the illustration; (3) require knowledge of end goals: an illustrator may not know a priori what degree of emphasis will achieve the intended result; and (4) lack intuitive interaction techniques and speed: to be able to create illustrations just as they envision them, illustrators need ease and freedom of interactive control and immediate visual response.

Inspired by professional illustrations and drawings, we developed a collection of tools called NPR Lenses to address these problems. To address the first problem, our NPR Lenses facilitate the application of both global and local effects. If desired, they can have a global effect but they can also be localized in a variety of different way such as: localized spatially, localized to chosen objects, localized to parts of objects, and even localized to specific sets of lines. Our solution allows illustrators to modify line properties locally by adjusting line shape, style, and presence independent from a scene-building object hierarchy. To address the second problem, our technique also provides visual integration between regions of emphasis thus avoiding visual discontinuities. To address the last two problems of freedom of intent and intuitive interaction, we integrated a 3D scene manipulation environment with $2 \mathrm{D}$ viewer-aligned lenses to support interactive creation of emphasis effects and dynamic exploration of the application of these effects. Our technique allows illustrators to explore line renderings of a 3D model by manipulating the view of a scene itself and to creatively play with the lenses and their effects until a desired illustration result is achieved.

The NPR Lenses approach differs from existing research and technology in the following ways: (1) NPR Lenses use 2D input to apply emphasis to line renditions that reside in a 3D rendering environment; (2) NPR Lenses allow artists to seamlessly apply a large variety of emphasis techniques, both locally and globally, independently of a scene hierarchy; (3) NPR Lenses all employ a common interaction approach, giving an illustrator freedom of chosen effects within a single interface; and (4) NPR Lenses support the creation of a variety of emphasis effects similar to those found in many existing hand-drawn illustrations.

The remainder of the article is structured as follows: We first discuss research related to NPR Lenses in Sec. 2. Sec. 3 describes the interaction framework on which NPR Lenses are based. Sec. 4 contains examples of expressive effects possible with NPR Lenses. We provide implementation details in Sec. 5 and a discussion of our approach in Sec. 6. Sec. 7 summarizes our contributions. 


\section{NPR Lenses in Context}

NPR Lenses relate to previous work in several research areas and, in particular, to interactive NPR stylization within a 3D graphics environment. We first introduce concepts and terminology that influenced our development of emphasis techniques for illustration and then discuss related research.

NPR Lenses are spatially explicit and constrained, view-dependent 3D scene adjustments. An adjustment to a 3D scene is spatially-explicit if the adjustment caused by the lens is located in the 3D world space. Thus, moving the model into or across the location of the lens affects the model accordingly. That means a single object may be partly within the adjustment space and partly not, thus allowing effects to be applied only to regions of a given object. A view-dependent adjustment is attached to the viewpoint and follows its movement in the 3D scene. A constrained adjustment has a region of influence that can be set to less than the full resulting image.

Fig. 1 shows different types of spatially explicit scene adjustments. One, the NPR Lens, extends "cone-like" from the viewpoint into the view frustum. Another lens, the Cube Lens as used in 3D Magic Lenses, exists at a specific location in the scene and influences objects in a cubed region in 3D [3]. The third example, the Half-Plane Lens, partitions the viewfrustum into an area of influence and one area that is not influenced [4]. All of these adjustments have a spatial explicit description and objects in the scene are either partially or fully in their influence ranges.

Spatially explicit emphasis is important for NPR line drawing since it is often seen in hand-drawn examples. Making NPR Lenses also viewer-aligned ensures that their effects are always visible. Related to this spatially explicit, viewer-aligned emphasis is the WYSIWYG-NPR system [5] that lets users add detail strokes and stylization directly to the scene from their view perspective. The primitives drawn on the image

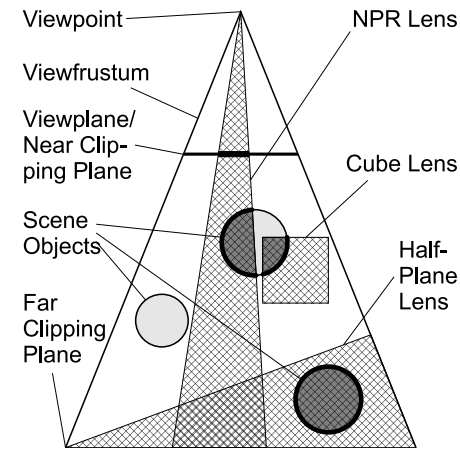

Fig. 1: Spatially explicit scene adjustments (crosshatched) in a $3 \mathrm{D}$ view frustum. Scene objects are partially in (darker) or out of their influence range. NPR Lenses are a subset of these and other possible spatially explicit scene adjustments. plane are projected onto the model and attached to it so that they remain on the surface when the model is rotated. This spatially explicit and view-dependent way to add detail only affects the top-most layer of the model similar to painting onto physical objects.

In NPR research, most stylization effects in 3D have been restricted to influence whole objects at a time. A popular technique for emphasizing model parts independently from the object structure is depth cueing, i. e., varying line attributes with respect to depth. Other examples that allow a much wider range of object-independent stylization effects include use of lighting conditions, viewing direction, specific axes in 3D space, or the relation to a $3 \mathrm{D}$ point of interest 
[4]. NPR Lenses add new possibilities to apply such object-independent emphasis. Our approach also relates to 3D Magic Lenses [3] where spatially explicit lenses are placed in a 3D virtual environment. This technique has been extended for real-time rendering and arbitrarily shaped spatially explicit lenses [6].

Spatial distortion is an emphasis technique that has received wide research attention but is usually not integrated with additional line stylization for creating emphasis. Morphing or warping are likely the most well-known techniques. These are commonly applied in image-space where pixels are shifted according to the manipulation of coordinate grid points or feature lines [7]. As an objectbased technique, spatial deformation is often used in animation to change the appearance of objects or transform one object into another [7]. Spatial distortion of the viewing space has been used for centuries in varying portrayals of depth such as in medieval art, renaissance art, or cubism. Recent viewing-space approaches in NPR distort scene geometry based on the manipulation of camera and viewing parameters to create expressive renditions $[8,9,10,11]$. While NPR Lenses incorporate spatial distortion, it is only one of the possible emphasis techniques. None of these require explicitly specified deformation functions or warping points. NPR Lenses, thus, have the added benefit of simple interaction and specification of a number of effects using one common interaction approach.

Our goal of making the NPR Lens interaction technique as familiar as the common interaction with 2D input devices such as mice or touch pads and successfully applying this to the manipulation of 3D scenes is a difficult problem. The difficulty results from correlation issues between the 2D mouse movement and object movement in the 3D virtual world [12]. Common 2D mouse interaction makes it difficult to exactly place or select an object in a $3 \mathrm{D}$ scene since the exact $z$-location of the cursor is not fully specified. Using a 3D input device to manipulate an object could help in specifying an exact $z$-location. However, the projection of the objects to the $2 \mathrm{D}$ screen and missing depth cues often makes it difficult to correctly analyze the position of 3D object parts and the 3D cursor in relation to each other.

\section{$3 \quad$ NPR Lens Interaction Framework}

We present an interaction framework that addresses 3D interaction difficulties by integrating 2D interaction and 3D application of effects. Our technique allows illustrators to interactively modify illustrations created by an otherwise fully automated line rendering process. The interaction has the feel of a $2 \mathrm{D}$ manipulation within a 3D graphics environment and provides the direct and expected 3D visual response. This interaction framework does not introduce the previously mentioned difficulties associated with interaction in 3D and achieves this without converting the $3 \mathrm{D}$ world to a $2 \mathrm{D}$ image. Thus, we can retain the advantages of $3 \mathrm{D}$ viewing, scene rotation, and line generation and provide simple interaction.

A metaphor will clarify how we integrate $2 \mathrm{D}$ and $3 \mathrm{D}$ interaction for NPR Lenses. Metaphorically, an NPR Lens parallels our use of a hand-held magnifying lens in the physical world. If you are examining a 3D physical scene with your magnifying lens you can move the lens freely over the scene or you can move 
objects in and out of your view. Your lens and scene are entirely independent but for the lens to be effective it must be placed between the eyes and the scene. NPR Lenses are similar and work on the same spatial principles but can achieve many different effects as well as magnification. The $3 \mathrm{D}$ rendering environment has a $3 \mathrm{D}$ scene that can be influenced by an NPR Lens. The lens operates on the viewplane and, like a physical magnifying lens, affects the entire portion of the $3 \mathrm{D}$ scene that is projected into its region of influence on the viewplane.

Since NPR Lenses integrate interaction in both $2 \mathrm{D}$ and $3 \mathrm{D}$ spaces, they have both a $2 \mathrm{D}$ and a $3 \mathrm{D}$ manifestation which is spatially explicit (Fig. 2). Their 2D manifestation on the viewplane (here shown on the near clipping plane) is defined by the lens' parameters. The 3D manifestation results from being projected from the viewplane into the $3 \mathrm{D}$ scene, called the lens frustum as in 3D Magic Lenses [3]. The lens frustum's shape is defined by the type of $3 \mathrm{D}$ projection and the chosen lens parameters (Fig. 2). In a perspective view frustum the NPR Lens is viewpoint-aligned whereas in an orthogonal view frustum the lens is view-direction-aligned. The lens parameters defined by the $2 \mathrm{D}$ manifestation ex-
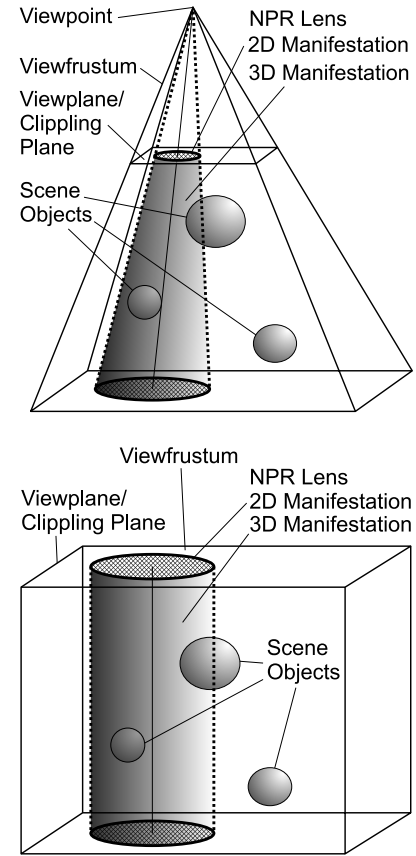

Fig. 2: Physical manifestation of NPR Lenses: perspective \& orthogonal frusta. tend consistently into the lens frustum according to the projection used.

Instead of having to adjust single scene entities, specify algorithms based on lighting or depth cues, or specify distortion functions to create emphasis effects we can freely position the NPR Lens' $2 \mathrm{D}$ manifestation on the viewplane and interactively manipulate it. Changing the location of the emphasis effects only requires moving the lens across the screen. This is independent of the objects in the scene and resembles moving a search light within a physical environment (Fig. 3). The view-alignment ensures that the lens provides the expected visual response as it will only affect objects within its lens frustum. The adjustments are applied in 3D in a process transparent to the users. Being

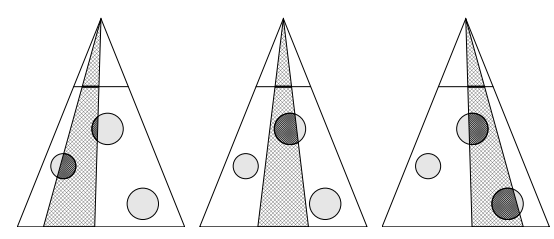

Fig. 3: Adjusting the 2D manifestation to move an NPR Lens in 3D. able to freely manipulate the viewpoint, users can place a lens on the screen and then rotate the model or camera to create the desired effects. Exploration of the model is possible and creative play with the lens and emphasis parameters allows for an interactive trial-and-error design process to achieve the final illustration.

We can also add any number of NPR Lenses to influence a 3D scene. Each lens can then be controlled and manipulated independently of the other lenses and the objects in the scene. When lenses intersect, both effects are integrated. 


\section{NPR Lens Effects}

The following describes several of the effects possible with NPR Lenses. Within the NPR Lens framework many other effects can easily be conceived and implemented. Here, we will describe Line-Style Lenses, Hidden-Line Lenses, SpatialDistortion Lenses, the application of object-based constraints, and the use of lenses in combination. It is important to notice that while an NPR Lens exists in $2 \mathrm{D}$ on the viewplane, its $3 \mathrm{D}$ manifestation, as projected into the $3 \mathrm{D}$ scene, operates on the entire set of 3D lines that have been extracted.

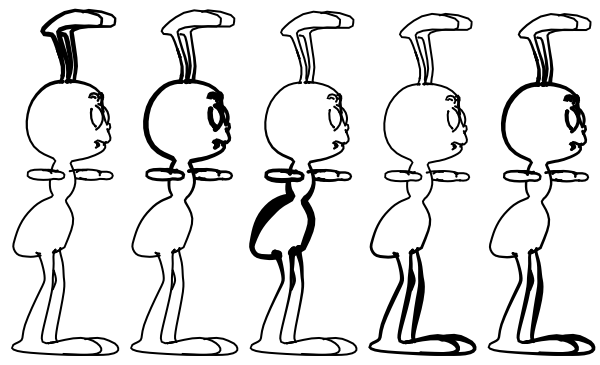

Fig. 4: Integrated local change of line style guides a viewer's attention.
The lenses have attributes such as a center, a type of effect, a degree of effect (DOE), a region of maximum effect, a region of influence, and an attenuation style. In designing a specific NPR Lens, one chooses lens attributes and what NPR line parameters to affect. The style of attenuation can be used, e.g., to create seamless integration from the affected region to those regions that are not. Alternatively, this factor can be ignored and an abrupt transition or distinct change from the lens region to the rest of the model may be created. While these factors are adjustable for all lenses, there are reasonable defaults that allow using lenses without constantly attending to such details. The interactivity of the lenses and their ability to provide seamless integration into the unmodified part of the image is a significant advantage of our method over previous object-independent stylization techniques.
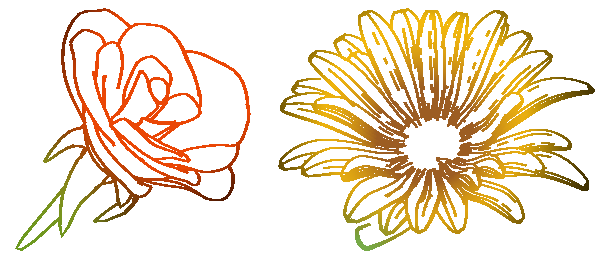

Fig. 5: Integrated local change of line color with two and three Line-Style lenses.

Line-Style Lenses can locally influence the line style of extracted strokes including aspects such as width, saturation, transparency, color, etc. In hand-drawn illustrations artists often use different line weights to place an emphasis on certain parts of an object and de-emphasize others. For example, varying line thickness can change the emphasis placed on particular aspects of the line renditions as shown in Fig. 4. Note how the emphasis on different body parts changes the impact of the images and can direct the attention of the viewer. This parallels effects described in [13,14] as being capable of influencing the viewer. Similarly, Fig. 5 shows how color can be used to highlight chosen aspects and to create localized lighting effects.

The Hidden-Lines Lens displays silhouette or feature lines of the underlying model that would not normally be visible from the current viewpoint and thus reveals otherwise hidden information. A distinct style such as a dotted line can be used to make these extra lines clearly distinguishable from regular lines (Fig. 6). 
This effect is similar to the wireframe lens mentioned as future work in the original magic lens paper [15] and similar to effects possible with 3D Magic Lenses [3]. In contrast to these techniques, we allow an individually adjustable views of hidden lines, blended into the surrounding context, while Ma-

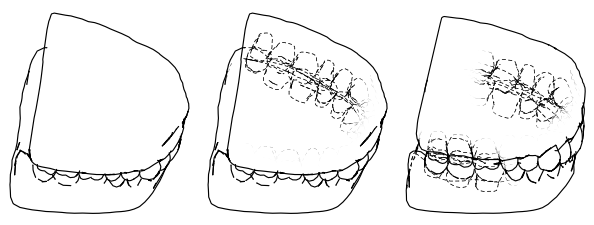

Fig. 6: None, one, and two Hidden-Line Lenses applied to a medical model. gic Lenses work as filters that have strong visual contrast at the edges of the lenses. One advantage of this blended filtered view of hidden lines - as opposed to always displaying them-lies in avoiding a cluttered display by showing hidden lines only where needed. The Hidden-Lines Lens can also be used to display other types of invisible lines, e.g., wireframe edges. The effects possible with such a lens are often seen in medical illustrations where objects behind the skin or other organs is made visible to guide learning in context.

The effects of a SpatialDistortion Lens resemble the effects created with other fisheye lenses. Such distortions can often be found in traditional hand-drawn illustrations where artists adjust chosen surface features or regions of particular importance to place an emphasis on aspects of an object's appearance.

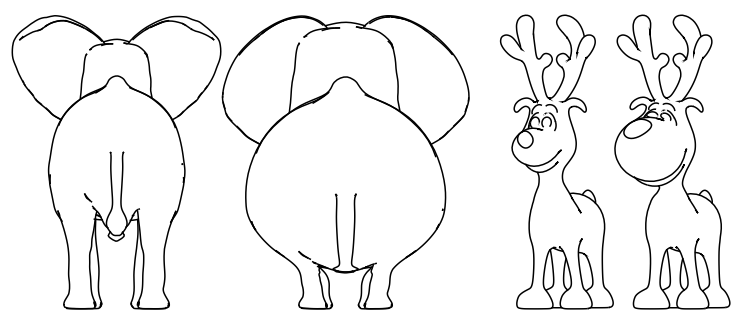

Fig. 7: Spatial distortion yields expressive line drawings (right; left: undistorted).

Also, carefully applied geometric distortions can give the resulting image a caricature or comic-like appearance. As such, the Spatial-Distortion Lens can be a valuable NPR tool. It allows to interactively change the appearance of line renditions to give them character according to one's intentions (Fig. 7). It can also be used as an interactive tool to examine aspects of an object that would otherwise remain unnoticed. In contrast to traditional zoom techniques, an examination of $3 \mathrm{D}$ model information is possible while still being able to view contextual information. Also, no modification of the model itself is necessary. An examination can take place via the lines created from these models.

Lenses as discussed so far have been applied to selected contiguous image regions. However, for any lens type, object-space constraints can be applied to restrict its application, to preserve some aspects of the model as unaffected or to choose to only affect par-

(a)

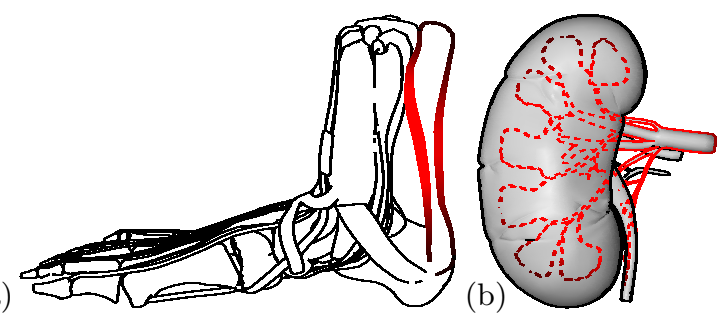

Fig. 8: Lens effect constrained in object-space. ticular parts of the model or selected objects. The objects to pass to the lens can 
be chosen in the graphical user interface. Fig. 8 shows two examples where the application of emphasis effects has been constrained: to the Achilles tendon of the foot in Fig. 8(a) and to the region of blood supply of the kidney in Fig. 8(b).

To improve the flexibility of the setup, all presented lenses as well as the object-space constraints can be combined in one lens manifestation. Fig. 8(a), e. g., demonstrates how a lens can influence both line color and thickness to emphasize the Achilles tendon of a foot. Here, a single lens in the interface applies several types of effects. Alternatively, one can choose to use several lens manifestations that each apply similar or different effects at different parts of the rendition as shown for line color in Fig. 5 and hidden lines in Fig. 6. Each of these manifestations can be individually controlled and parameterized.

\section{Implementation}

\begin{tabular}{|c|c|c|}
\hline $\begin{array}{l}\text { Silhouette } \\
\text { Detection }\end{array}$ & $\begin{array}{c}\text { Feature Edge } \\
\text { Detection }\end{array}$ & $\begin{array}{c}\text { Stroke } \\
\text { Concatenation }\end{array}$ \\
\hline$\sqrt{5}$ & & $\tau$ \\
\hline $\begin{array}{l}\text { Hidden Line } \\
\text { Removal }\end{array}$ & $\begin{array}{l}\text { Stroke } \\
\text { Stylization }\end{array}$ & $\begin{array}{c}\text { Stroke } \\
\text { Rendering }\end{array}$ \\
\hline
\end{tabular}

Fig. 9: Traditional pipeline.
To generate line renderings from polygonal models we use the OPENNPAR framework [16]. The line rendering process is modeled in a pipeline consisting of a series of rendering nodes. These nodes manage vertex coordinates and the edge data connecting these vertices (Fig. 9). The pipeline takes a 3D model as input and first extracts silhouettes and feature lines. Next, these lines are concatenated to longer strokes and hidden line data is stored for each vertex. Stroke stylization determines a line's rendering style including texture, thicknesses, and color. The pipeline may contain optional steps to, e.g., remove artifacts or to add parameterization data (e.g., object IDs) to later modify the strokes' appearance. All stroke coordinates are kept in 3D to render them onto the underlying model.

NPR Lenses are used to manipulate line rendering styles. This can be done coherently by using $G$-strokes [17]. This concept separates geometry information from stylization parameters, allowing pipeline nodes to specify and change stylization parameters for line segments. G-strokes are used in our work to capture the line style parameters' changes applied using lenses.

NPR Lenses are also based on the EPF framework [18] which places a 2D data representation onto a base plane that itself is located in the $x$-y-plane in $3 \mathrm{D}$ space. The base plane is viewed from above, perspectively projected. Different presentation styles can be created by manipulating the base plane. These manipulations (EPF Lenses) are functions that can be parameterized to produce the desired effects. We use this flexible and fast means to specify and compute 2D emphasis effects and, in particular, the fact that the DOE is known for any location within the lens, making it possible to affect factors other than location.

To apply lens techniques to line drawings rendered from 3D models we have to adjust the traditional rendering pipeline (Fig. 9). In the first step, as usual, silhouettes or feature lines are extracted from the model. Next, these lines are concatenated to form long stokes and their visibility is determined. At this point the line data is available for the lens to modify. To add these new steps, NPR Lens nodes are inserted into the pipeline. They are applied before the line stylization 
and rendering stage so that the effects of the lens can be reflected in these two stages (Fig. 10). With this setup each lens takes geometric information as input and influences styl-

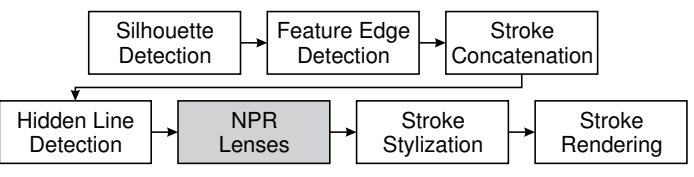

Fig. 10: Modified line rendering pipeline. ization and rendering of the lines. Each NPR Lens node introduced in the line rendering pipeline comprises four steps: (1) take a $3 \mathrm{D}$ input Point $P(x, y, z)$ from the list of calculated silhouette or feature line points, $(2)$ project $P$ to image-space yielding $P(w x, w y, w z),(3)$ obtain values from an EPF lens using $w x$ and $w y$ yielding a new point $P^{\prime}\left(w x^{\prime}, w y^{\prime}, w z\right)$ and a degree of effect factor doe for this point, and (4) use all or just a subset of these values obtained from $\mathrm{EPF}$ as input for a specific NPR Lens.

This sequence represents a hybrid image- and object-space approach. To allow illustrators to apply emphasis effects as if a lens would be moved over a $2 \mathrm{D}$ line rendition, the emphasis effect has to be applied in $2 \mathrm{D}$ coordinates. Hence, each $3 \mathrm{D}$ vertex is first projected to image-space where the emphasis is applied. Then, the vertices are back-projected into 3D world-space coordinate. We need to maintain the world coordinate stroke pipeline because some further stroke processing requires world coordinates. The doe and new coordinate information is then used by an NPR Lens as input for modifying the style of the given line rendition to create expressive variations or to allow exploration of the model.

The computational impact of this additional pipeline step is small since only the extracted strokes have to be processed. Thus, its rendering time depends on the complexity of the processed lines and not that of the model from which the lines are derived. In fact, the bottleneck of the stroke pipeline usually lies in the silhouette edge extraction and hidden line removal and not the following stroke processing. We found that meshes suitable for line rendering (i. e., not producing too many lines that would overly clutter the image) are rendered at interactive to real-time frame rates. Thus, NPR Lenses typically do not impact the system's interactivity; only for very detailed strokes with several lenses applied the lens computation is noticeable but interactive frame rates are still maintained.

The NPR Line-Style Lens uses only the degree of effect doe to control the line style parameter by multiplying or adding doe to the previous value, resulting in changing the line style parameter's G-stroke. Adding doe creates more subtle effects than multiplying. The extent of the desired influence is user-controlled through setting the usual lens parameters. Similarly, we use the computed doe value to reveal hidden model information within the influence range of the lens by only processing vertices that have a raised doe value. For these vertices we now render otherwise hidden layers of the image such as hidden lines, either on top of the regular visible lines or instead of them. The new layers are smoothly blended into the surrounding context according to the doe to achieve visual continuity and can be given a certain (e.g., dotted) line style.

For creating a Spatial-Distortion Lens the 3D positions of the stroke vertices obtained from the geometric model are projected to image-space and subjected 
to the spatial distortion as described above. The discrete, object-space nature of this processing may introduce artifacts since the topology of the strokes is

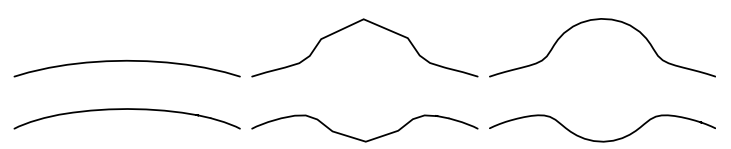

(a) Undistorted. (b) Discrete. (c) Continuous. not modified: large doe factors result in the stepwise linear character of edges to become visible (Fig. 11(b)). This ef-

Fig. 11: Addressing the artifacts from strong discrete distortion (b) with adaptive subdivision (c).

fect could be avoided by working with parametric curves between vertex positions. This in turn may result in the lines deviating too much from the expected object shape and being visually awkward. Real continuous distortion, on the other hand, is computationally impossible. It can only be emulated by using a dense set of vertices in the original strokes. This, however, increases the required storage capacity and slows down processing. Moreover, a dense vertex sampling is only required in regions with high doe values. Thus, we use adaptive stroke subdivision to approximate continuous distortion in magnified regions (Fig. 11(c)). This keeps the vertex density roughly constant and the number of additional vertices at the necessary minimum.

Object-space constraints are applied during the feature and silhouette line generation. An ID parameter is generated for each vertex that indicates from which object in the scene or model it was extracted. Based on this ID value, filtering can be applied in the NPR Lens node and only objects that are passed through the filter will be influenced by the lenses.

\section{Discussion and Future Work}

We suggested a number of example domains such as medical illustration (Fig. 8 and 12(a)), technical illustration (Fig. 12(b)), or even caricature (Fig. 7) where NPR Lenses can be used to create expressive effects. While we have introduced

(a)

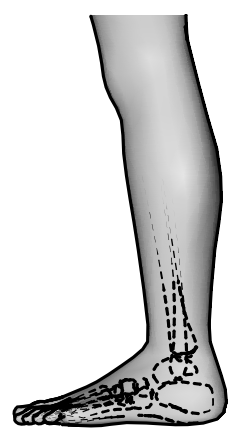

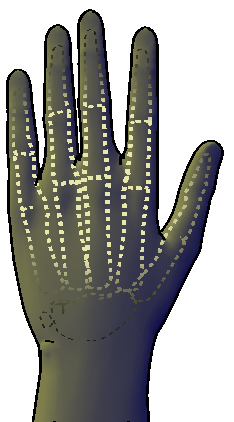

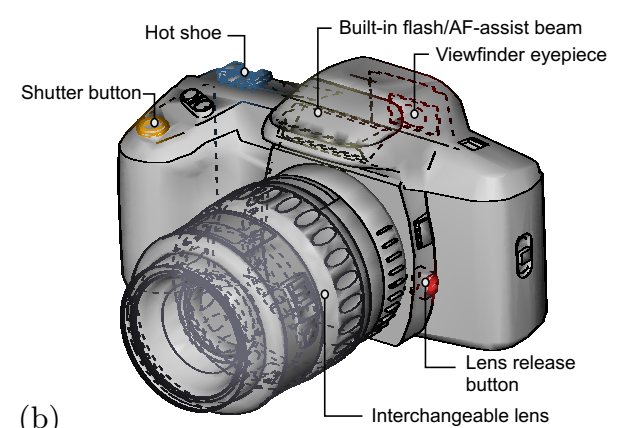

(b)

Fig. 12: Medical (a) and technical illustrations (b) using lens combinations and background shading, the latter with added annotations. Components are highlighted using color lenses and components inside the camera are shown using the Hidden-Lines Lens. 
and discussed several different types of NPR Lenses, many other effects are possible with our framework. Possible other lenses could include the manipulation of line transparency or texture and the application to other line types such as hatching or stippling. Lenses could be used as brushes to add lines where desired or as erasers to remove or hide lines where they are not wanted.

NPR Lenses were built to be a useful tool for the creation of static illustrations for print-reproduction or use in electronic media. They are better suited for creating still images rather than line drawing animations. When the $3 \mathrm{D}$ scene is moved under a placed NPR Lens, the effects resulting from that lens do not rotate with the model but affect the new parts of the model in the influence range of the lens. This is a direct result of our spatially explicit scene adjustment approach. Making effects stay on the the objects is one possible direction of future work. Another potential direction is to apply emphasis not only to extracted lines but to the underlying mesh as well by applying a distortion to the mesh vertices and moving them in 3D space while keeping the mesh's topology. Our lenses could provide an intuitive 2D interaction method for polygonal modeling that applies effects in 3D. Preliminary results are promising (Fig. 13).

Although we have not yet formally evaluated NPR Lenses, they have been tried by students and a profes-

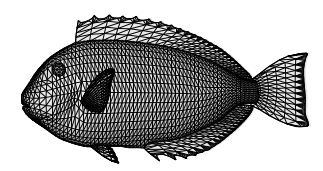

(a) Undistorted mesh.

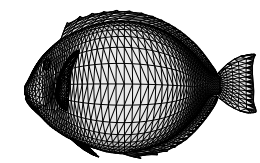

(b) One distortion step.

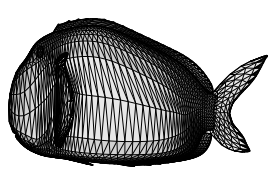

(c) Several iterations.

Fig. 13: Mesh distortion with NPR Lenses. sional artist. Generally, they found the tool easy to use and enjoyed adjusting and distorting the rendered line drawings. Often one person using the tool would attract attention from others and instigate more usage. Future work will include more thorough studies to assess how useful our tool is to, e. g., professional illustrators and what features they require for their work.

\section{Conclusion}

This paper introduces the NPR Lens interaction framework built for an easy and intuitive creation of expressive line renderings for illustration. The emphasis techniques provided in our framework are used frequently in art and illustration as a means to express emotional content, guide the user's attention, or articulate an artist's or illustrator's view of the depicted scene. NPR Lenses were developed with the intent of providing illustrators with a tool that allows for creative freedom in the illustration process. This is achieved by using 2D lens controls inside the 3D rendering pipeline, thereby integrating the advantages of 3D scene manipulation with the capabilities of spatially-explicit, viewer-aligned lenses.

NPR Lenses make four main contributions: (1) they integrate 2D input and 3D application of effects to make the application of emphasis effects with NPR Lenses as simple as the common interactions with a mouse, touchpad, or stylus; (2) they allow the seamless application of emphasis effects both locally and globally independent of a scene hierarchy; (3) they provide a common interaction 
approach, thereby, allowing a creative exploration of the application of effects without a priori knowledge of end goals; and (4) they support the creation of a variety of emphasis effects common in hand-drawn illustration in a single extensible interface. We hope that this research will spark new interest in the creation of NPR tools targeted at the illustration community, so that NPR techniques will be used more frequently and successfully in the future.

\section{References}

1. Gooch, B., Gooch, A.: Non-Photorealistic Rendering. A K Peters, Natick (2001)

2. Strothotte, T., Schlechtweg, S.: Non-Photorealistic Computer Graphics. Modelling, Animation, and Rendering. Morgan Kaufmann Publishers, San Francisco (2002)

3. Viega, J., Conway, M.J., Williams, G., Pausch, R.: 3D Magic Lenses. In: Proc. of UIST 96, New York, ACM Press (1996) 51-58

4. Isenberg, T., Masuch, M., Strothotte, T.: 3D Illustrative Effects for Animating Line Drawings. In: Proc. of IV 2000, Los Alamitos, IEEE (2000) 413-418

5. Kalnins, R.D., Markosian, L., Meier, B.J., Kowalski, M.A., Lee, J.C., Davidson, P.L., Webb, M., Hughes, J.F., Finkelstein, A.: WYSIWYG NPR: Drawing Strokes Directly on 3D Models. ACM Transactions on Graphics 21(3) (July 2002) 755-762

6. Ropinski, T., Hinrichs, K.: Real-Time Rendering of 3D Magic Lenses Having Arbitrary Convex Shapes. Journal of WSCG 12(1-3) (2004) 379-386

7. Parent, R.: Computer Animation Algorithms and Techniques. Morgan Kaufmann Publishers, San Francisco (2002)

8. Rademacher, P.: View-Dependent Geometry. In: Proc. of SIGGRAPH 99, New York, ACM Press (1999) 439-446

9. Martín, D., García, S., Torres, J.C.: Observer Dependent Deformations in Illustration. In: Proc. of NPAR 2000, New York, ACM Press (2000) 75-82

10. Singh, K.: A Fresh Perspective. In: Proc. of GI 2002, Natick, A K Peters (2002) $17-24$

11. Coleman, P., Singh, K.: RYAN: Rendering Your Animation Nonlinearly Projected. In: Proc. of NPAR 2004, New York, ACM Press (2004) 129-138

12. Foley, J.D., van Dam, A., Feiner, S.K., Hughes, J.F.: Computer Graphics: Principles and Practice in C. $2^{\text {nd }}$ edn. Addison-Wesley, Reading (1990)

13. Strothotte, T., Preim, B., Raab, A., Schumann, J., Forsey, D.R.: How to Render Frames and Influence People. Computer Graphics Forum 13(3) (1994) 455-466

14. Winkenbach, G.A., Salesin, D.H.: Computer-Generated Pen-and-Ink Illustration. In: Proc. of SIGGRAPH 94, New York, ACM Press (1994) 91-100

15. Bier, E.A., Stone, M.C., Pier, K., Buxton, W., DeRose, T.D.: Toolglass and Magic Lenses: The See-Through Interface. In: Proc. of SIGGRAPH 93, New York, ACM Press (1993) 73-80

16. Halper, N., Isenberg, T., Ritter, F., Freudenberg, B., Meruvia, O., Schlechtweg, S., Strothotte, T.: OpenNPAR: A System for Developing, Programming, and Designing Non-Photorealistic Animation and Rendering. In: Proc. of Pacific Graphics 2003, Los Alamitos, IEEE Computer Society (2003) 424-428

17. Isenberg, T., Brennecke, A.: G-Strokes: A Concept for Simplifying Line Stylization. Computers \& Graphics 30(5) (October 2006) 754-766

18. Carpendale, S., Montagnese, C.: A Framework for Unifying Presentation Space. In: Proc. of UIST 2001, New York, ACM Press (2001) 61-70 